\title{
A dynamic model for road protection against flooding
}

\author{
Stefano Starita*a, M. Paola Scaparra ${ }^{\mathrm{b}}$, and Jesse R. O'Hanley ${ }^{\mathrm{b}}$ \\ ${ }^{\mathrm{a}}$ Warwick Business School, University of Warwick, Coventry CV4 7AL, UK \\ ${ }^{\mathrm{b}}$ Kent Business School, University of Kent, Canterbury CT2 7PE, UK
}

\begin{abstract}
This paper focuses on the problem of identifying optimal protection strategies to reduce the impact of flooding on a road network. We propose a dynamic mixed-integer programming model that extends the classic concept of road network protection by shifting away from single-arc fortifications to a more general and realistic approach involving protection plans that cover multiple components. We also consider multiple disruption scenarios of varying magnitude. To efficiently solve large problem instances, we introduce a customised GRASP heuristic. Finally, we provide some analysis and insights from a case study of the Hertfordshire road network in the East of England. Results show that optimal protection strategies mainly involve safeguarding against flooding events that are small and likely to occur, whereas implementing higher protection standards are not considered cost-effective.
\end{abstract}

Keywords: road transportation; flooding; network disruption; infrastructure protection; MILP; GRASP

\section{Introduction}

Natural hazards can have serious impacts in terms of economic losses and human casualties. Floods can be particularly dangerous due to the high number of people living in at-risk areas and due to their relatively high frequency of occurrence. Both coastal and inland areas can be affected by floods caused by the overflow of rivers and the sea. Even areas that are far from a water course or coastal zone can still be at risk of flooding caused by heavy rain. The

\footnotetext{
${ }^{*}$ Correspondence email: s.starita@kent.ac.uk
} 
World Resources Institute estimates that climate change and population rise will more than double (from 21 to 54 million) the number of people exposed to floods (Luo et al., 2015). In the UK, one in six properties is at risk of flooding (Environmental Agency, 2009). The 2005 flood in New Orleans caused by Hurricane Katrina is one of the most tragic examples of the disruptive power of such events. Even less dramatic floods can still lead to major disruption of vital services and cause significant economic losses. During the winter of 2013-14 in the UK, for example, heavy rainfall triggered numerous floods that left major roadways under water and disrupted train lines. The government subsequently allocated $£ 130 \mathrm{M}$ to repair and maintain flood defences (Carrington and Weaver, 2014).

The first step to mitigate flood risk has to be done at the policy-level, so as to regulate urban development in hazardous areas. Subsequently, risk analysis should be implemented to identify vulnerabilities and suitable protection measures. Resources should also be used to devise post-disaster strategies to reduce loss of life and economic damages.

Flood events are usually classified using the "return period" concept (Gumbel, 1941). The return period is the time interval between two events of the same disruption size and can be used to estimate the probability of a given scenario occurring. A 100 year flood, for instance, it is estimated to happen with a $1 \%$ chance in any given year.

The same concept is also used to define the standard of protection offered by an engineered flood defence measure. Common types of flood defence include building/replacing dikes, embankments, slopes, sluices, and culverts. These structures differ in terms of the standard of protection guaranteed, the cost to implement them, and the size of area afforded protection. Relatively inexpensive defences like vertical walls or concrete structures can often be quickly built to protect a small, targeted area. More ambitious plans, such as the construction of diversion canals or large dams, can impact larger areas, but normally at a much higher cost.

Flood damages can be broadly split into three categories: urban, rural, and infrastructure (Dutta et al., 2003). In this paper, we deal with the issue of protecting a road transportation system. Transportation systems are often highly exposed to flood risk due to their considerable size, which makes implementing fully comprehensive protection measures all but impossible. In order to cope with the limited availability of resources for securing road networks, we propose a multi-period optimisation model for selecting multiple asset protection plans to guard against a range of potential flood scenarios. Solving the proposed model to optimality requires a substantial amount of computational effort. Accordingly, we devise a heuristic solution approach to efficiently tackle realistic sized problem instances. 


\section{Literature review}

The problem of protecting critical infrastructure has been increasingly studied over the last decade, especially in the context of facility location. A popular stream of literature focuses on minimizing the impact of a worst-case scenario. This approach is particularly suited to situations involving an intelligent adversary (e.g., military strikes or terrorist attacks) but can still prove useful when dealing with large, catastrophic natural disasters (e.g., earthquakes and hurricanes). Church and Scaparra (2007) study the problem of finding the optimal set of facilities to protect in a supply chain system that is subject to disruption. Losada et al. (2012) introduce the concept of recovery time into the facility protection problem. Aliakbarian et al. (2015) extend the problem of protecting a supply chain system by considering hierarchical facilities. Alguacil et al. (2014) study a model to find the best allocation of defensive or hardening resources in an electric grid subject to intentional disruption.

The majority of work relating to transportation has focused on identifying system vulnerabilities (i.e., using interdiction type models) rather than finding the best set of elements to protect (i.e., using fortification-interdiction type models). One exception is Starita and Scaparra (2015), who study the problem of protecting railway infrastructure over a planning horizon through the use of flow networks. Likewise, Liberatore et al. (2011) consider the problem of protecting facilities against worst-case attacks when the number of disrupted elements is uncertain. Cappanera and Scaparra (2011) propose a model to identify the set of elements to harden so as to minimise the shortest path between two nodes. Alderson et al. (2011) investigate the problem of protecting bridges and tunnels of a transportation system in the face of terrorist attacks. Jin et al. (2015), meanwhile, introduce a tri-level model for the protection of urban rail transit networks. Sarhadi et al. (2015) focus on rail inter-modal transportation, studying a fortification model that embeds a capacitated multi-commodity flow problem with delivery times and penalty costs.

Protecting infrastructure is a complex problem in which numerous factors may be uncertain. Worst-case models can be overly pessimistic, especially when dealing with natural events that are more random in nature. To tackle this issue, a second important line of research focuses on the development of stochastic programming models. Berman et al. (2007), for example, optimise the location of unreliable facilities in a p-median network. They propose exact and heuristic approaches for the situation in which facilities have site-dependent failure probabilities. Zhang et al. (2014) propose a model to protect supply systems when facilities are subject to random attacks. They also introduce a hybrid model aimed at finding optimal protection strategies for both random and worst-case attacks.

Disruption of transportation and supply networks have been dealt with by various authors. 
Viswanath et al. (2004) study the problem of distributing resources to reduce the expected shortest path of a network subject to independent random failures. Investments are made on links to increase their survival probability. Du and Peeta (2014) introduce a two-stage stochastic model which aims to reduce the expected post-disaster response time by distributing pre-disaster resources among the links of a shortest-path network. Rawls and Turnquist (2010) study emergency response planning for hurricanes and other natural disasters. A model is proposed to determine the quantity, type, and location of supplies to be pre-positioned, while taking into account the availability of transportation links following a disruption event. Fan and Liu (2010) investigate the protection of transportation networks against disruption. Their model is formulated as a stochastic network optimisation problems with equilibrium constraints that takes into account repair cost, travel delay cost, and unsatisfied demand. Liu et al. (2009) propose a two-stage stochastic model to optimally retrofit a multi-commodity flow network. The aim is to fortify a subset of highway bridges so that the transportation system's resiliency is maximised according to a mean-risk objective. Madadi et al. (2014) also examine the importance of risk attitude in the context of designing reliable supply chain networks by comparing risk-neutral and risk-averse decision criteria.

A third distinct line of research looking at facility and network disruption involves the use of robust optimisation. Here, the aim is to identify solutions that perform well across a defined set of disruption scenarios. Often times, information about the likelihood of disruption events is lacking or of lesser importance. Rather, scenarios are chosen based mainly on the severity of impact they can have on system efficiency. This is often the case when planning against large natural disasters.

Among the group of robust optimisation studies, Huang et al. (2007) develop a maximum covering model for the optimal allocation of emergency vehicles in a transportation network. Scenarios are included to handle variations in travel times and demand and a regret type metric is used to evaluate the robustness of allocation strategies. Yin (2008) investigate the problem of finding the optimal allocation of tow trucks so as to improve response times and minimise disruptions to traffic flow. Scenarios correspond to different incident levels occurring in the network and are used to form a conditional value-at-risk measure. Current et al. (1998) consider a dynamic facility location problem where the number to open facilities is uncertain. The goal of the problem is to minimise expected opportunity loss and minimise maximum regret. Snyder and Daskin (2006) propose a robustness measure for facility location problems called p-robustness. Their model seeks to minimise the expected cost of facility disruptions, while bounding the relative regret of each scenario. Chen et al. (2006) introduce the $\alpha$-reliable mean-excess risk measure. The model they propose minimises expected regret, focusing on an endogenously selected subset of scenarios. Peng et al. (2011) propose a model to design 
reliable supply chain networks. The aim is to minimise cost when no disruption occurs and reduce the risk of disruptions using the $p$-robustness measure. Rawls and Turnquist (2012) extend Rawls and Turnquist (2010) by incorporating a regret-based reliability metric and illustrate their approach using a case study hurricanes threatening the North Carolina area. Ben-Tal et al. (2011) develop a robust optimisation framework for a dynamic traffic assignment problem with demand uncertainty. The framework is applied to an emergency response and logistic planning test problem. Najafi et al. (2013) propose a multi-objective robust optimisation model to efficiently use scarce emergency resources in the aftermath of an earthquake. Baghalian et al. (2013) introduce a robust model for designing supply chains when demand is uncertain and manufacturers are subject to disruptions.

Other studies dealing with infrastructure disruption that do not fit neatly within the afore mentioned categories include Görmez et al. (2011), who study the problem of locating relief centers in anticipation of a large earthquake. A sensitivity analysis is carried out to understand how solutions perform under various disruption scenarios. Jozefowiez et al. (2012) propose a shortest-path based heuristic for rescheduling airlines over a multi-period horizon when disruptions occur. Ishfaq (2013) formulate a shortest-path model which considers inter-modal shipments to react to transportation link disruptions.

Focusing specifically on road transportation system vulnerability and protection, Ukkusuri and Yushimito (2009) propose a heuristic based on user equilibrium flows to assess the criticality of highway links. Faturechi and Miller-Hooks (2014) study a stochastic model with equilibrium constraints to optimise travel time in a roadway network subject to disruption. He and Liu (2012) propose a prediction-correction model to evaluate day-to-day traffic flow evolution following a road network disruption. Chang (2003) develop a performance measure based on accessibility to evaluate the functionality of an urban transportation system in the aftermath of a disaster. Peeta et al. (2010) study a shortest-path based model to decide which links to strengthen in a highway network that are subject to random failures.

Much less attention has been given to the effects of flooding on transportation services. Suarez et al. (2005) propose a model to assess how flooding and climate change impact the performance of urban transportation. They use an urban transportation modelling system (UTMS) to simulate traffic flows and apply the model to the Boston metropolitan area. Sohn (2006) assess the critical links in a highway network under flood damage. Criticality is estimated according to an accessibility metric that integrates shortest path distances and traffic flows. 


\section{Model Formulation}

\subsection{Problem description and assumptions}

Let a road network be represented as a graph $G(N, A)$, where $N$ is the set of nodes (junctions) and $A$ the set of arcs (road links). Suppose a set of flood disruption scenarios $S$ is under consideration. A given flood disruption scenario $s \in S$, which occurs with probability $\pi_{s}$, will cause a subset of arcs $A_{s} \subseteq A$ to be disrupted. The disruption caused by any scenario $s$ can vary spatially such that arcs may face a range of discrete flood magnitudes represented by set $L$. The return period of a given flood size $\ell \in L$ is given by $r_{\ell}$ and expresses the average time interval (normally expressed in years) between two floods of the same magnitude. A flood's magnitudes and return period are directly linked. Specifically, flood events with longer return periods have higher magnitude and vice versa. Set $R=\left\{r_{\ell}: \ell \in L\right\}$ denotes all possible return periods associated with flood sizes $L$.

Under normal conditions, travel time along an arc $k \in A$ is given by $d_{k}$. Assuming no additional protection is provided to an arc, flood scenario $s \in S$ will cause a delay in travel time of $\Delta d_{k s}$ for arc $k \in A_{s}$ as a result of facing a flooding event with a return period of $\rho_{k s} \in R$. We assume that protection of any arc $k$ to a standard sufficient to withstand a flood of magnitude $\ell \in L$ will protect it against any flood of lesser magnitude (i.e., no delay will occur for any flood $\ell^{\prime} \in L$ having a return period $\left.r_{\ell^{\prime}}<r_{\ell}\right)$. With this in mind, let $P$ be a set of protection plans. An individual protection plan $p \in P$, which $\operatorname{costs} c_{p}$ to implement, can protect multiple arcs $\hat{A}_{p} \subseteq A$ to various standards. Specifically, plan $p$ will protect arc $k \in \hat{A}_{p}$ against any flood with a return period of $\sigma_{k p} \in R$ or less.

Now the aim of our problem is to select a subset of protection plans in $P$ over a specified planning horizon of length $T$ in order to minimise, across disruption scenarios $S$, the expected all-pairs shortest path from a defined origin $o \in N$. Each time period is subject to the same set of scenarios $S$. It is assumed that there is a budget $b_{t}$ available in each period for implementing protection plans and that unused portions of the budget can be carried forward to subsequent periods. It is further assumed that protection of any arc in time $t=1, \ldots, T$ lasts for the remainder of the planning horizon $t, \ldots, T$. In cases where two or more protection plans are implemented and would provide overlapping protection to the same arc, the arc will be protected to the highest standard among the plans (e.g., if an arc has already been protected to a standard $\ell$ and a subsequent plan would protect it to a lower standard $\ell^{\prime}$ such that $r_{\ell^{\prime}}<r_{\ell}$, then the previous higher standard $\ell$ would be retained). 


\subsection{A Dynamic model for Road Protection against Flooding}

To formulate our Dynamic Road Protection against Flooding (DRPF) model, consider the following additional notation:

$$
\begin{aligned}
& F S(i)=\text { the forward star of node } i \text { (i.e., the set of arcs directed out of node } i \text { ) } \\
& R S(i)=\text { the reverse star of node } i \text { (i.e., the set of arcs directed into node } i \text { ) } \\
& M=\text { a large constant greater than or equal to the largest return period in set } R
\end{aligned}
$$

We also introduce the following decision variables:

$y_{k s t}^{o}=$ the number of times arc $k$ is included in a shortest path originating from node $o$ to any other node if scenario $s$ occurs in period $t$

$$
\begin{aligned}
& z_{p t}= \begin{cases}1 & \text { if plan } p \text { is implemented in period } t \\
0 & \text { otherwise }\end{cases} \\
& \alpha_{k s t}= \begin{cases}1 & \text { if } \operatorname{arc} k \text { is disrupted by scenario } s \text { in period } t \\
0 & \text { otherwise }\end{cases}
\end{aligned}
$$

$\beta_{k t}=$ the largest return period that $\operatorname{arc} k$ would be protected against in period $t$

With this in place, a non-linear formulation of DRPF is given below.

$$
[\mathrm{DRPF}] \quad \min \sum_{s \in S} \pi_{s} \sum_{t=1}^{T} \sum_{o \in N} \sum_{k \in A}\left(d_{k} y_{k s t}^{o}+\Delta d_{k s} \alpha_{k s t} y_{k s t}^{o}\right)
$$

s.t.

$$
\begin{aligned}
& \sum_{u=1}^{t} \sum_{p \in P} c_{p} z_{p u} \leq \sum_{u=1}^{t} b_{u} \quad t=1, \ldots, T \\
& \sum_{k \in R S(i)} y_{k s t}^{o}-\sum_{k \in F S(i)} y_{k s t}^{o}= \begin{cases}-|N|+1 & \text { if } i=0 \\
1 & \text { otherwise } \\
\beta_{k t}=\max _{u \leq t, p \in P}\left\{\sigma_{k p} z_{p u}\right\} & \forall k \in N, \forall i \in N, \forall s \in S, t=1, \ldots, T\end{cases}
\end{aligned}
$$




$$
\begin{aligned}
& M \alpha_{k s t} \geq \rho_{k s}-\beta_{k t} \quad \forall k \in A, \forall s \in S, t=1, \ldots, T \\
& y_{k s t}^{o} \geq 0 \quad \forall o \in N, \forall k \in A, \forall s \in S, t=1, \ldots, T \\
& z_{p t} \in\{0,1\} \quad \forall p \in P, t=1, \ldots, T \\
& \alpha_{k s t} \in\{0,1\} \quad \forall k \in A, \forall s \in S, t=1, \ldots, T \\
& \beta_{k t} \in \mathbb{R} \quad \forall k \in A, \forall t=1, \ldots, T .
\end{aligned}
$$

The aim of DRPF model is to choose protection plans that minimise the expected all-pairs shortest path cost over all disruption scenarios (1). Inequalities (2) impose a budget restriction on the cost of protection plans in each time period $t$. Equations (3) are the flow-balance constraints for an all-pairs shortest-path problem. Equations (4) set $\beta_{k t}$ to the largest return period that arc $k$ would be protected against taking into account all of the protection plans covering arc $k$ that have been implemented in periods $u \leq t$. Constraints (5) require variable $\alpha_{k s t}$ to be equal to 1 whenever the highest safety standard implemented for arc $k$ by period $t$ is insufficient to protect against scenario $s$ (i.e., if $\rho_{k s}>\beta_{k t}$ ). Finally, constraints (6)-(9) impose necessary the restrictions on the decision variables.

\subsection{Linearisation of the objective function}

The second term of the objective function $\Delta d_{k} \alpha_{k s t} y_{k s t}^{o}$ is non-linear. To linearise it, we introduce auxiliary variables $\gamma_{k s t}^{o}$ as well as a large constant $M^{\prime}$. Objective function (1) can then be replaced by:

$$
\min \sum_{s \in S} \pi_{s} \sum_{t=1}^{T} \sum_{k \in M} \sum_{o \in N}\left(d_{k} y_{k s t}^{o}+\Delta d_{k} \gamma_{k s t}^{o}\right)
$$

subject to (2)-(9) and the following.

$$
\begin{aligned}
& \gamma_{k s t}^{o} \leq M^{\prime} \alpha_{k s t} \quad \forall o \in N, \forall k \in A, \forall s \in S, t=1, \ldots, T \\
& \gamma_{k s t}^{o} \geq y_{k s t}^{o}+M^{\prime}\left(\alpha_{k s t}-1\right) \quad \forall o \in N, \forall k \in A, \forall s \in S, t=1, \ldots, T \\
& \gamma_{k s t}^{o} \geq 0 \quad \forall o \in N, \forall k \in A, \forall s \in S, t=1, \ldots, T
\end{aligned}
$$

Constraints (11) and (12) are introduced so that $\gamma_{k s t}^{o}=y_{k s t}^{o}$ whenever $\alpha_{k s t}=1,0$ otherwise. Constant $M^{\prime}$ needs to be greater than or equal to the total number of origin-destination pairs. Constraints (13) are the non-negativity requirements for the $\gamma_{k s t}^{o}$ variables. 


\subsection{Linearisation of the protection level constraints}

Constraints (4) can be linearised by introducing additional decision variables $x_{k t \ell}$ defined as:

$$
x_{k t \ell}= \begin{cases}1 \quad \text { if the highest protection standard afforded to arc } k \text { in period } t \\ \text { is sufficient to withstand a flood of size } \ell \\ 0 \quad \text { otherwise }\end{cases}
$$

Further, let $P_{k \ell}$ be the subset of plans in $P$ that would protect arc $k$ to a standard sufficient to withstand a flood of size $\ell$. With this in place, equalities (4) can be replaced with the following set of linear constraints.

$$
\begin{aligned}
& \beta_{k t}=\sum_{\ell \in L} r_{\ell} x_{k t \ell} \quad \forall k \in A, t=1, \ldots, T \\
& \sum_{\ell \in L} x_{k t \ell} \leq 1 \quad \forall k \in A, t=1, \ldots, T \\
& x_{k t \ell} \leq \sum_{u=1}^{t} \sum_{p \in P_{k \ell}} z_{p u} \quad \forall k \in A, \forall \ell \in L, t=1, \ldots, T \\
& x_{k t \ell} \in\{0,1\} \quad \forall k \in A, t=1, \ldots, T
\end{aligned}
$$

Constraints (14) are used to set $\beta_{k t}$ to the highest protection standard implemented for arc $k$ by period $t$. Inequalities (15) stipulate arc $k$ can be protected to at most one safety standard $\ell$ in period $t$. Constraints (16) state that arc $k$ can be protected to a safety standard of $\ell$ in period $t$ if a plan $p \in P_{k \ell}$ has been implemented in the time window $1, \ldots, t$. Constraints (17) are the binary requirements for variables $x_{k t \ell}$.

\section{Solution approach}

The proposed model can be solved directly with a general purpose mixed integer linear programming (MILP) solver like CPLEX. The formulation, however, requires a very large number of variables and constraints, even when a moderate number of scenarios, protection plans, and time periods is considered. This has a strong impact on the performance of the MILP solver, both in terms of computing time and memory requirements. Moreover, shortest-path problems can be solved very efficiently using specialised algorithms. These considerations suggest that more effective solution approaches can be devised. Here, we 
present a heuristic algorithm based on the GRASP (Greedy Randomised Adaptive Search Procedure) meta-heuristic for building an initial solution combined with a local search to further explore the feasible space.

\subsection{GRASP step}

GRASP was introduced by Feo and Resende (1995) to overcome the limits of purely greedy construction algorithms. It is an iterative procedure that at each step builds up a solution by randomly choosing elements from a dynamically constructed restricted candidate list (RCL). The RCL consists of a subset of elements which are selected according to a greedy scheme. Once GRASP has produced a starting solution, a local search can be carried out to find an improved solution. The entire process can be repeated multiple times in an effort to more fully explore the solution space.

The following is the notation used in our GRASP implementation:

- itr is the iteration index

- plansitr and $o b j_{i t r}$ are the solution and corresponding objective value obtained at iteration itr

- bestPlans and bestObj refer to the best solution found and corresponding objective value

- MAXITER is the maximum number of iterations

- RCLSIZE is the size of the restricted candidate list

- $\Phi_{p t}$ is a greedy metric used to rank the pair $(p, t)$ (i.e., the importance of plan $p$ implemented at time $t$ )

$-S_{k \ell}$ is the set of scenarios that disrupt arc $k$ at level $\ell$

$-\Delta_{s}$ is the increment in the all-pairs shortest path expected cost generated by disruption scenario $s$

We further introduce some crucial subroutines used in the algorithm:

- computeShortestPath(plans $\left.s_{i t r}\right)$ finds a solution, with protection strategy plans $s_{i t r}$ as input, to the all-pairs shortest path problem subject to the disruption scenarios. The function is implemented efficiently using the Floyd-Warshall algorithm (Floyd, 1962). 
- updateImportance $\left(\right.$ plans $\left._{i t}\right)$ updates the metric $\Phi_{p t}$ given plans $s_{i t}$ as input. Before defining $\Phi_{p t}$ mathematically, we need to introduce an additional parameter $\lambda_{k \ell}$ for each arc and flood size pair $(k, \ell)$. Specifically, let $\lambda_{k \ell}=\sum_{\ell^{\prime} \leq \ell} \sum_{s \in S_{k \ell}} \Delta_{s}$. Parameter $\lambda_{k \ell}$ gives the cumulative increment in the shortest path expected travel cost generated by all scenarios disrupting arc $k$ at level $\ell^{\prime} \leq \ell$. For the sake of clarity, we define $f i t=$ computeShortestPath(plans $\left.s_{i t r}\right)$ and $f i t(p, t)=$ computeShortestPath $\left(\right.$ plans $\left._{i t r} \cup(p, t)\right)$.

The difference between these two values, $f i t-f i t(p, t)$, represents the net decrease in overall travel costs obtained when adding $(p, t)$ to the solution. The importance metric is then computed by the following formula:

$$
\Phi_{p t}=\frac{\sum_{k \in \hat{A}_{p}} \lambda_{k \sigma_{k p}}(f i t-f i t(p, t))}{c_{p}}
$$

Looking at (18), importance is defined as the benefit obtained by including plan $p$ at time $t$ to the set of implemented protections weighted by parameter $\lambda_{k \sigma_{k p}}$ and the inverse of the cost $c_{p}$ of implementing that plan.

- buildRCL(plans $\left.s_{i t r}\right)$ creates the restricted candidate list of size $R C L S I Z E$ by selecting the best pairs $(p, t)$ according to their $\Phi_{p t}$ values.

- updateFeasibePlans(plansitr) is used to keep track of plans that can be added to plans $_{\text {itr }}$ without violating the budget constraints.

In Algorithm 1, we provide pseudo-code of the GRASP heuristic.

\subsection{Improvements to the GRASP algorithm}

Here, we briefly discuss some expedients that have been adopted to improve the GRASP algorithm both in terms of efficiency and accuracy. The first improvement takes advantage of multi-core processors by implementing a multi-thread version of the algorithm. Using concurrent programming allows for a more in-depth exploration of the solution space without any increase in execution time. Furthermore, threads can work with different algorithm settings to implement different policies so that the probability of exploring the same solution is reduced. To provide this flexibility, we extend the definition of $\Phi_{p t}$ as follows:

$$
\Phi_{p t}=\frac{\mu(p) \sum_{k \in \hat{A}_{p}} \lambda_{k \sigma_{p}}(f i t-f i t(p, t))}{c_{p}}
$$




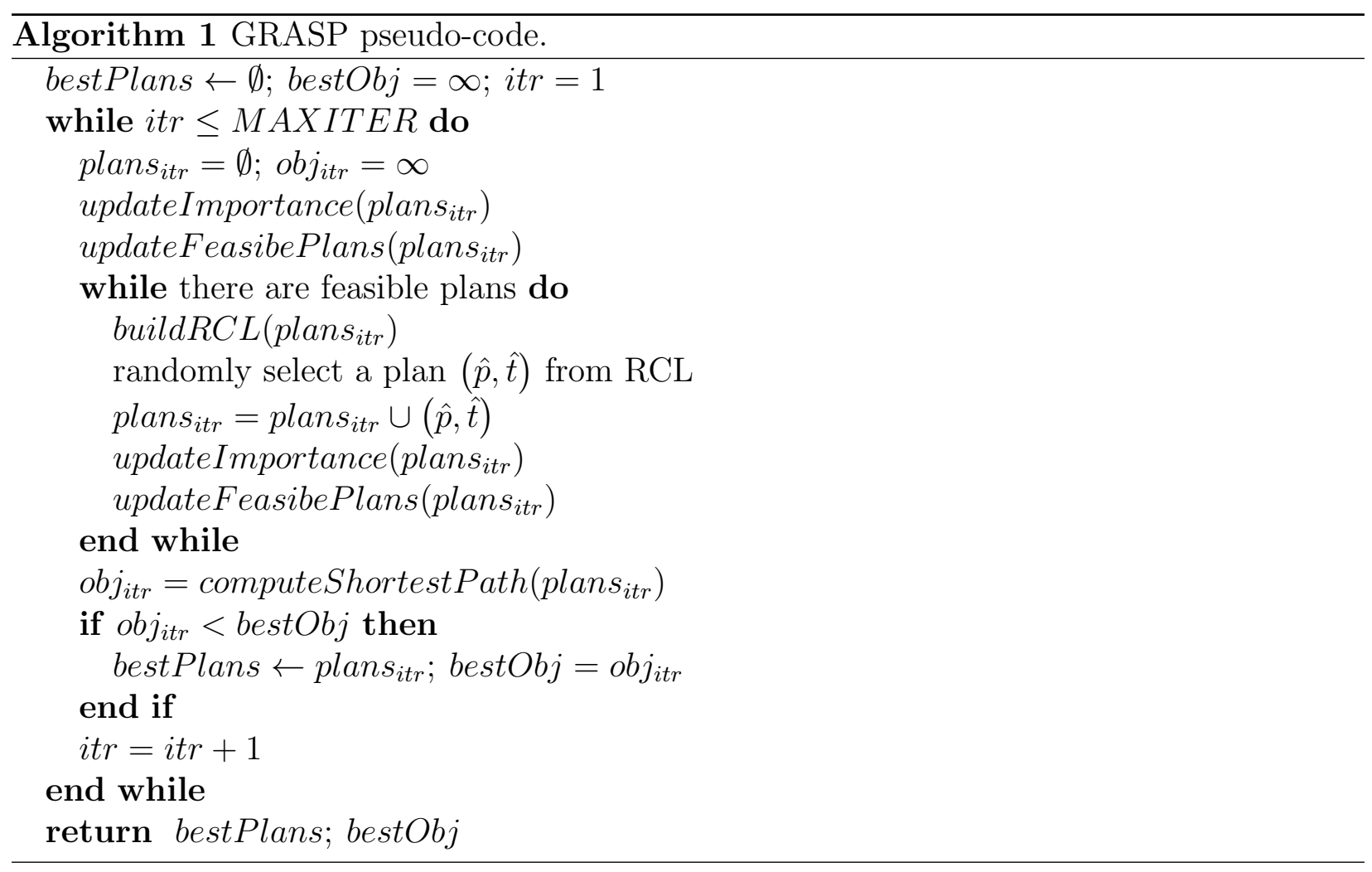

The function $\mu(p)$ is used to weight each plan according to the protection level offered. Specifying different functional forms of $\mu$ for each thread will result in different solutions being explored, thus increasing the chance of finding an optimal solution. For example, $\mu(p)=1$ weights all plans $p$ equally. Alternatively, $\mu(p)=\ell_{p}^{\max }$, where $\ell_{p}^{\text {max }}$ is the index number of the maximum protection standard afforded to any arc in plan $p$ (i.e., $\ell_{p}^{\max }=\arg \max _{k \in \hat{A}_{p}} \sigma_{k p}$ ), puts higher weight on plans offering potentially higher levels of protection. This, in turn, increases the probability of having such plans in the solutions.

The algortihm can be further improved by optimizing the routine updateImportance(). The idea behind the improvement is as follows. If there are available protection resources at time $t$, the most important plans (the ones with higher $\Phi)$ will more likely be the plans $\left(p, t^{\prime}\right)$ with $t^{\prime} \in[t, t+1]$. As a consequence, we can reduce the number of plans for which $\Phi$ needs to be recomputed by excluding the plans $\left(p, t^{\prime}\right)$ such that $t^{\prime} \in[t+2, T]$.

Finally, if we keep track of the highest possible level of disruption threatening each arc, we can ignore any plans that overprotect. Formally, a plan $p$ is excluded from consideration if:

$$
\begin{aligned}
\sigma_{k p}>\max _{s \in S}\left\{\rho_{k s}\right\} \forall k \in \hat{A}_{p} \wedge \exists p^{\prime} \text { s.t. } c_{p}^{\prime}<c_{p}, \hat{A}_{p}^{\prime} \subseteq \hat{A}_{p} \\
\sigma_{k p} \geq \sigma_{k p^{\prime}} \geq \max _{s \in S}\left\{\rho_{k s}\right\} \forall k \in \hat{A}_{p^{\prime}}
\end{aligned}
$$




\subsection{Local search step}

The best solution found by the GRASP procedure is used as the starting point for a local search. The temporal component of the model makes the implementation of an effective local search challenging. In fact, a search procedure should take into account the fact that the GRASP solution generated might include plans that are not included in the optimal solution and/or plans that are in the optimal solution but should be implemented at different time periods. To tackle this issue, we implemented two types of swap moves: internal and external. For each type, two approaches were devised. The four different swaps are carried out sequentially in the same order as they are introduced in the next sections.

\subsubsection{Internal swap}

Internal swaps can perform single or multiple swaps between the elements of a solution. Specifically, these swaps change only the time when plans are implemented, not the type of plans chosen. Swaps are done only among plans implemented in consecutive time periods. We consider two combinations of swaps:

- 1-to-2 swap: A plan $(p, t)$ with $t \in[1, T-1]$ is postponed one period. Simultaneously, up to two plans implemented at time $t+1$ are brought forward one period. Every combination involving 1 or 2 plans is considered. Non-feasible solutions are discarded. The pseudo-code for this move is shown in Algorithm 2.

- 2-to-3 swap: This move is almost identical to the previous one. The only difference is the number of plans that are postponed (2) and the number that are brought forward (up to 3$)$.

Table 1 shows an example of a 1-to-2 internal swap, where the plans in bold are those being swapped. It is worth mentioning that the code has been implemented to avoid unnecessary computation. For instance, assuming that the swap $p_{2} \leftrightarrow\left(p_{3}, p_{5}\right)$ generates a feasible solution, then swaps $p_{2} \leftrightarrow p_{3}$ and $p_{2} \leftrightarrow p_{5}$ are not considered.

\begin{tabular}{|c|c|c|c|c|c|c|c|}
\hline \multicolumn{4}{|c|}{ Before } & \multicolumn{4}{|c|}{ After } \\
\hline$t$ & & lan & & $t$ & & Plan & \\
\hline 0 & $p_{1}$ & $p_{2}$ & & 0 & $p_{1}$ & $p_{3}$ & $p_{5}$ \\
\hline 1 & $p_{3}$ & $p_{4}$ & $p_{5}$ & 1 & $p_{2}$ & $p_{4}$ & \\
\hline 2 & $p_{6}$ & $p_{7}$ & & 2 & $p_{6}$ & $p_{7}$ & \\
\hline
\end{tabular}

Table 1: Example of 1-to-2 internal swap. 


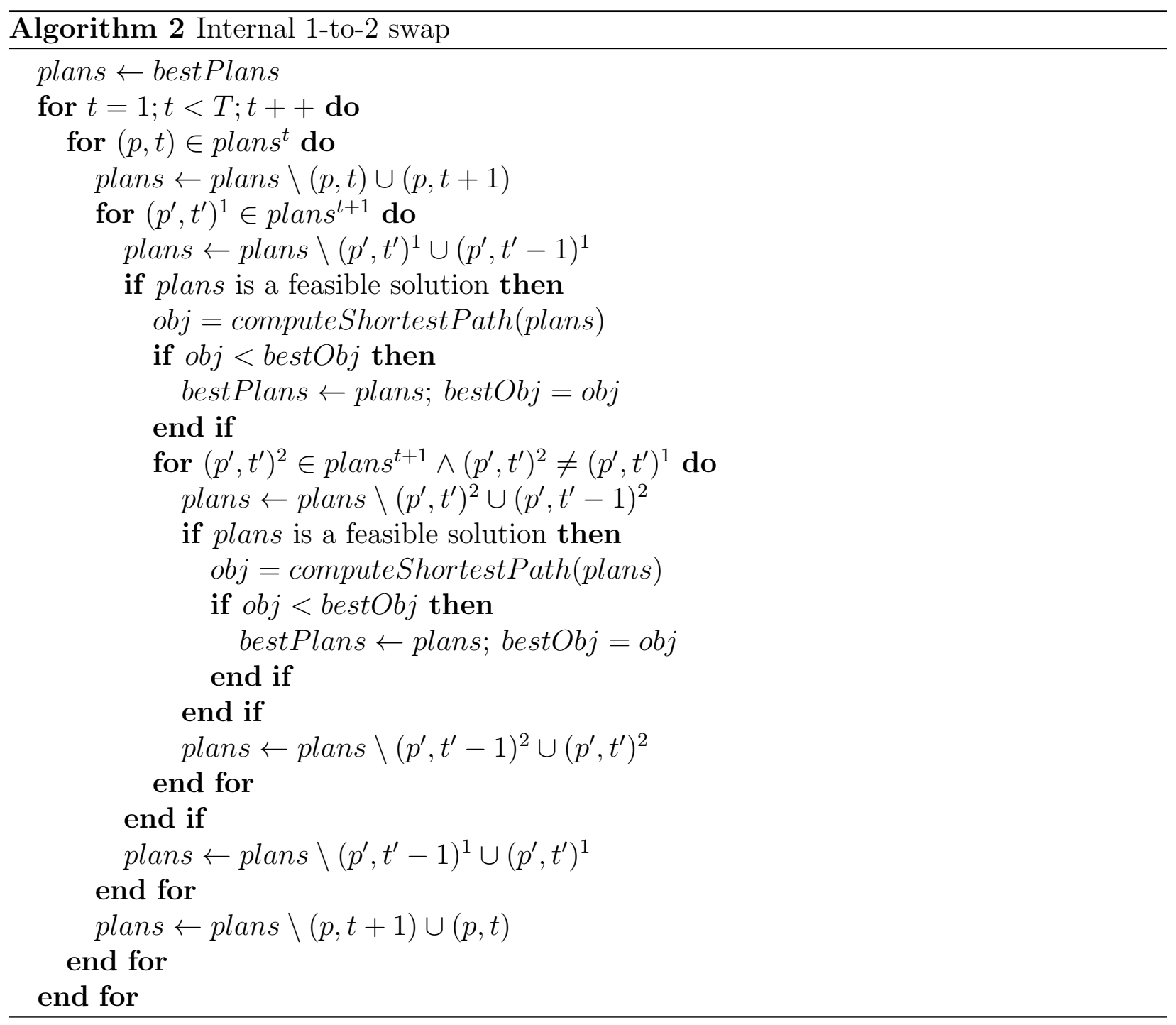

\subsubsection{External swap}

External swaps remove one or more plans from the incumbent solution and replace them with one or more plans not currently included in the solution. As with internal swaps, we consider two cases:

- 1-to-2 swap: A plan $(p, t)$ is removed from the solution. Simultaneously, up to two plans are added in its place. Only plans that would generate a feasible solution are considered. The pseudo-code for this move is shown in Algorithm 3.

- 2-to-3 swap: This move is nearly identical to the previous one. The only difference is the number of plans swapped out (2) and swapped in (up to 3). 


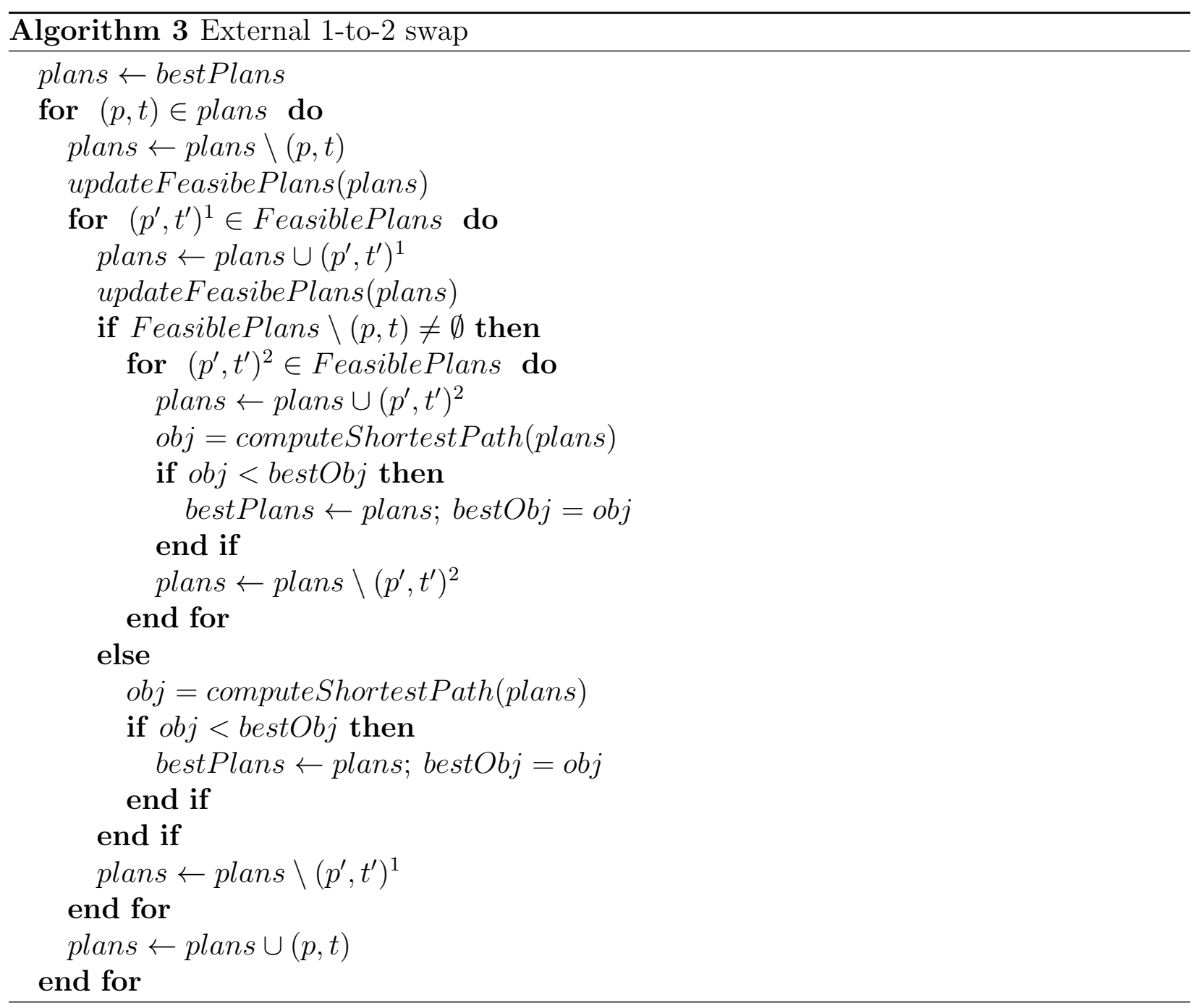

Table 2 shows an example of 1-to-2 external swap with swapped plans shown in bold.

Before

After

\begin{tabular}{|c|c|c|c|c|c|c|c|c|c|c|c|c|}
\hline$t$ & & ernal & plans & \multicolumn{3}{|c|}{ External plans } & $t$ & \multicolumn{3}{|c|}{ Internal plans } & \multicolumn{2}{|c|}{ External plans } \\
\hline 0 & $p_{1}$ & & \multirow{3}{*}{$p_{5}$} & $p_{8}$ & $\overline{p_{9}}$ & $p_{10}$ & 0 & $p_{1}$ & $p_{2}$ & & $p_{7}$ & $p_{8}$ \\
\hline 1 & $p_{3}$ & $p_{4}$ & & $p_{8}$ & $p_{9}$ & $p_{10}$ & 1 & $p_{3}$ & $p_{4}$ & $p_{5}$ & $p_{7}$ & $p_{8}$ \\
\hline 2 & $p_{6}$ & $p_{7}$ & & $p_{8}$ & $p_{9}$ & $p_{10}$ & 2 & $p_{6}$ & $p_{9}$ & $p_{10}$ & $p_{7}$ & $p_{8}$ \\
\hline
\end{tabular}

Table 2: Example of 1-to-2 external swap.

\section{Computational tests}

For our initial computational tests, we generated undirected square networks of size $n \times m$, where $n$ is the number of nodes and $m$ is the number of arcs. We assume that the travel time 


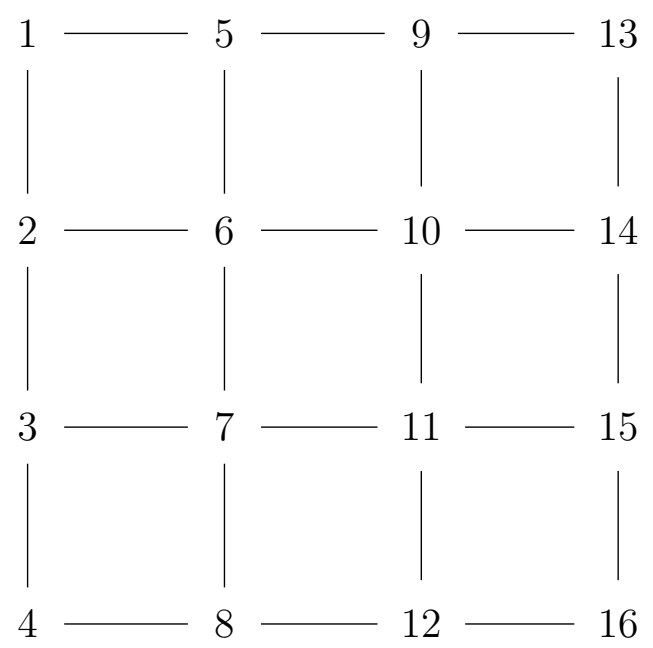

Figure 1: An example $16 \times 24$ grid.

along a link is independent of travel direction and that protections and disruptions affect simultaneously and equally both directions. In Figure 1, we show the topology of an example $16 \times 24$ grid. Arcs travel times $d_{k}$ were drawn uniformly in the range $[1,10]$.

We consider 4 different flood sizes, categorised in terms of their return period: 20, 50, 100 , and 250 years. The same values are used to identify levels of protection, hence $R=$ $\{20,50,100,250\}$. According to the disruption level of each scenario, the delay $\Delta d_{k s}$ is set to $2.5,5,10$, and 20 times the baseline travel time $d_{k}$. We examined three types of protection plans: arc, node, and row/column plans. Arc plans protect a single arc. Node plans protect all the arcs incident to a node. Finally, row/column plans include all the arcs forming a row or a column in the grid. For example, if we consider the grid in Figure 1, the node plan corresponding to node 6 includes arcs $2-6,5-6,6-7$, and $6-10$. The row plan corresponding to the second row includes arcs 2-6, 6-10, and 10-14. Overall, we can identify $m+3 n$ different plans using this scheme. Each plan can be implemented at 4 levels of protection. Consequently, for each instance we use $4(m+3 n)$ protection plans. In Table 3, we show the criteria used to estimate the costs of implementing the protection plans.

The discount factor $f$ was set to 1.3. It is incorporated so that adopting a node or row-column

\begin{tabular}{lcccc}
\hline & \multicolumn{4}{c}{ Return period (yrs) } \\
\cline { 2 - 5 } Type of plan & 20 & 50 & 100 & 250 \\
\hline Arc & $d_{k}$ & $2 d_{k}$ & $3 d_{k}$ & $4 d_{k}$ \\
Node & {$\left[f^{-1} \sum_{k \in \hat{A}_{p}} c_{k}\right]$} & {$\left[2 f^{-1} \sum_{k \in \hat{A}_{p}} c_{k}\right]$} & {$\left[3 f^{-1} \sum_{k \in \hat{A}_{p}} c_{k}\right]$} & {$\left[4 f^{-1} \sum_{k \in \hat{A}_{p}} c_{k}\right]$} \\
Row/Column & {$\left[f^{-1} \sum_{k \in \hat{A}_{p}} c_{k}\right]$} & {$\left[2 f^{-1} \sum_{k \in \hat{A}_{p}} c_{k}\right]$} & {$\left[3 f^{-1} \sum_{k \in \hat{A}_{p}} c_{k}\right]$} & {$\left[4 f^{-1} \sum_{k \in \hat{A}_{p}} c_{k}\right]$} \\
\hline
\end{tabular}

Table 3: Cost of implementing a given type of plan according to its protection level. 
plan $p$ will be cheaper than independently protecting all the arcs included in $p$.

We assume that all arcs face the same level of disruption for any given flood scenario (i.e., $\left.\rho_{k s}=\rho_{s}, \forall k \in A_{s}\right)$ and that the number of arcs disrupted in each scenario is dependent on the scenario's return period. The least damaging scenario is capable of disrupting only 1 arc. The number of disrupted arcs increases with the scenario's size from 1 to $m / 4$ (i.e., the most severe disruption scenario can disrupt $25 \%$ of the network). In Table 4 , we list the number of disrupted arcs for each of the return periods considered.

\begin{tabular}{lcccc}
\hline & \multicolumn{3}{c}{ Return period (yrs) } \\
\cline { 2 - 5 } & 20 & 50 & 100 & 250 \\
\hline No. disrupted arcs & 1 & $\lceil(m+20) / 24\rceil$ & $\lceil(m+5) / 9\rceil$ & $\lceil m / 4\rceil$ \\
\hline
\end{tabular}

Table 4: Number of disrupted arcs based on a scenario's return period.

Scenarios were built in such a way that arcs affected by a given scenario formed a connected sub-network. This was done so that the elements affected by a flood event were contiguous, as is often the case in real life. In Table 5, we report the number of scenarios generated for each level of flooding. The probability of occurrence for a scenario was set as the inverse of the scenario's return period. We generated 30 instances using networks of size $9 \times 12,16 \times 24$, and $25 \times 40$.

\begin{tabular}{|c|c|c|c|c|}
\hline & \multicolumn{4}{|c|}{ Return period (yrs) } \\
\hline & 20 & 50 & 100 & 250 \\
\hline No. scenarios & $m$ & $\bar{m} / 2\rceil$ & $m / 4\rceil$ & $m / 81$ \\
\hline
\end{tabular}

Table 5: Number of scenarios generated for each return period.

We considered a 4-period planning horizon (i.e., $T=4$ ). We chose a total protection budget equal to $10 \%$ of the resources needed to protect the entire network at the highest safety level. The budget was equally spread over the time periods. We ran the heuristic with the number of threads set to 4 . Each thread uses a different $\mu$ vector to compute $\Phi$ based on equation (19). The values for the different policies used in each thread are listed in Table 6.

\begin{tabular}{ccccc}
\hline & \multicolumn{4}{c}{ Return period (yrs) } \\
\cline { 2 - 5 } Thread ID & 20 & 50 & 100 & 250 \\
\hline 0 & 1 & 1 & 1 & 1 \\
1 & 1 & 2 & 3 & 4 \\
2 & 1 & 1.3 & 1.6 & 1.9 \\
3 & 1 & 2 & 2 & 1 \\
\hline
\end{tabular}

Table 6: Set of $\mu$ values used for each thread. 


\begin{tabular}{|c|c|c|c|c|c|c|c|}
\hline \multirow[b]{2}{*}{ Network size } & \multirow[b]{2}{*}{ Instance } & \multicolumn{2}{|c|}{ CPLEX } & \multicolumn{4}{|c|}{ Heuristic } \\
\hline & & Obj & Time(s) & Obj & Time(s) & $\operatorname{Gap}(\%)$ & Time diff(\%) \\
\hline \multirow[t]{11}{*}{$9 \times 12$} & 1 & 2168.6 & 7 & 2168.6 & 2.5 & $0.00 \%$ & $-64.29 \%$ \\
\hline & 2 & 2595.5 & 6.9 & 2595.5 & 3.3 & $0.00 \%$ & $-52.17 \%$ \\
\hline & 3 & 2282.4 & 9.7 & 2282.4 & 3.8 & $0.00 \%$ & $-60.82 \%$ \\
\hline & 4 & 2831.9 & 6.5 & 2831.9 & 2.9 & $0.00 \%$ & $-55.38 \%$ \\
\hline & 5 & 2476.4 & 4.7 & 2476.4 & 3.1 & $0.00 \%$ & $-34.04 \%$ \\
\hline & 6 & 3107.9 & 6.6 & 3107.9 & 3.3 & $0.00 \%$ & $-50.00 \%$ \\
\hline & 7 & 2360.5 & 8.8 & 2360.5 & 3.8 & $0.00 \%$ & $-56.82 \%$ \\
\hline & 8 & 2600.1 & 6.9 & 2600.1 & 3.6 & $0.00 \%$ & $-47.83 \%$ \\
\hline & 9 & 2505.0 & 5.6 & 2505.0 & 3.3 & $0.00 \%$ & $-41.07 \%$ \\
\hline & 10 & 1755.5 & 8.9 & 1756.1 & 2.7 & $0.03 \%$ & $-69.66 \%$ \\
\hline & Avg & & 7.2 & & 3.2 & $0.00 \%$ & $-53.21 \%$ \\
\hline \multirow[t]{11}{*}{$16 \times 24$} & 1 & 17020.9 & 1900.1 & 17020.9 & 176.9 & $0.00 \%$ & $-90.69 \%$ \\
\hline & 2 & 19487.3 & 3581.8 & 19487.3 & 164.7 & $0.00 \%$ & $-95.40 \%$ \\
\hline & 3 & 21347.0 & 1714.5 & 21347.0 & 131.6 & $0.00 \%$ & $-92.32 \%$ \\
\hline & 4 & 16744.5 & 1553.9 & 16747.5 & 166.6 & $0.02 \%$ & $-89.28 \%$ \\
\hline & 5 & 18646.8 & 1354.2 & 18646.8 & 129.4 & $0.00 \%$ & $-90.44 \%$ \\
\hline & 6 & 16661.8 & 4395.2 & 16662.7 & 187.8 & $0.01 \%$ & $-95.73 \%$ \\
\hline & 7 & 15346.2 & 4322.8 & 15349.1 & 135.1 & $0.02 \%$ & $-96.87 \%$ \\
\hline & 8 & 14288.9 & 387.1 & 14288.9 & 207.4 & $0.00 \%$ & $-46.42 \%$ \\
\hline & 9 & 18783.4 & 2177.7 & 18783.4 & 192.2 & $0.00 \%$ & $-91.17 \%$ \\
\hline & 10 & 12225.7 & 1971.92 & 12225.7 & 180.9 & $0.00 \%$ & $-90.83 \%$ \\
\hline & Avg & & 2335.9 & & 167.3 & $0.00 \%$ & $-87.92 \%$ \\
\hline \multirow[t]{11}{*}{$25 \times 40$} & 1 & $106278.9^{\dagger}$ & 21600.0 & 106064.0 & 3183.7 & $-0.20 \%$ & $-85.26 \%$ \\
\hline & 2 & $88124.5^{\dagger}$ & 21600.0 & 87981.4 & 3665.6 & $-0.16 \%$ & $-83.03 \%$ \\
\hline & 3 & $97700.2^{\dagger}$ & 21600.0 & 97684.1 & 3676.1 & $-0.02 \%$ & $-82.98 \%$ \\
\hline & 4 & $91102.4^{\dagger}$ & 21600.0 & 91025.2 & 3448.5 & $-0.08 \%$ & $-84.03 \%$ \\
\hline & 5 & $109276.9^{\dagger}$ & 21600.0 & 109091.0 & 3876.9 & $-0.17 \%$ & $-82.05 \%$ \\
\hline & 6 & $80102.2^{\dagger}$ & 21600.0 & 79972.0 & 4384.8 & $-0.16 \%$ & $-79.70 \%$ \\
\hline & 7 & $88891.3^{\dagger}$ & 21600.0 & 88729.7 & 4102.1 & $-0.18 \%$ & $-81.01 \%$ \\
\hline & 8 & $97849.8^{\dagger}$ & 21600.0 & 97818.4 & 4315.6 & $-0.03 \%$ & $-80.02 \%$ \\
\hline & 9 & $115763.9^{\dagger}$ & 21600.0 & 115681.0 & 3937.2 & $-0.07 \%$ & $-81.77 \%$ \\
\hline & 10 & $83557.7^{\dagger}$ & 21600.0 & 83530.9 & 3988.9 & $-0.03 \%$ & $-81.53 \%$ \\
\hline & Avg & & 21600.0 & & 3857.9 & $-0.11 \%$ & $-82.14 \%$ \\
\hline
\end{tabular}

${ }^{\dagger}$ Best objective value found after 6 hours of running time.

Table 7: Computational results for test problem instances.

Thread 0 treats all the protection plans equally. Threads 1 and 2 give more importance to plans that grant higher levels of protection. Finally, thread 4 weights more heavily medium level protection plans. These values were chosen empirically to maximise the probability of discovering an optimal solution. The size of the RCL is dependent on the problem size. It was set to 3 for $9 \times 12$ and $16 \times 24$ networks and to 4 for $25 \times 40$ networks. The number of iterations was set to 100 and we specified a 6-hour limit on the run time of the algorithm.

In Table 7, we compare the performance of CPLEX against our GRASP heuristic on the 30 
test problem instances. The "Obj" and "Time" columns show the objective value and the solution time obtained by the two algorithms. "Gap" is the percentage gap between the best feasible solution obtained with CPLEX and the value returned by the heuristic. A negative value for "Gap" indicates that the heuristic found a better solution. Finally, "Time diff" is the percentage difference between the computing times of the two algorithms. A negative value for "Time diff" indicates that the heuristic was faster than CPLEX. The table shows that CPLEX can find a verified optimal solution within 6 hours only for small and mediums sized instances. In fact, on the $25 \times 40$ networks, CPLEX never converges to an optimal solution. The heuristic, on the other hand, finds optimal or near-optimal solutions much more efficiently. It is on average at least $82 \%$ faster than CPLEX and always returns better solutions for the $25 \times 40$ networks.

\section{Case study}

In this section, we present a case study based on the A-road network infrastructure of Hertfordshire in the UK. The network is composed of 36 nodes and 47 undirected arcs. We used historical data on floods, which are publicly available through the Environmental Agency (EA). Using geographic information system (GIS) software (ArcGIS), we generated disruption scenarios by identifying regions where floods and the road network overlapped. Over the past 70 years, 29 floods in Hertfordshire are recorded in the EA database. Among them, 16 overlapped with the A-road network.

In Figure 2, we show the road network considered in our case study. Recorded floods are shown in light blue; in dark blue are shown events that affected the A-road network. Unfortunately, floods are not categorised in the database. Consequently, we allocated them into three size categories (small, medium, and large) according to the extent of the area flooded. The corresponding return periods for each flood size is shown in Table 8. Coming up with more precise estimates for return periods and, consequently, the likelihood of each flood category requires a detailed hydrogeological analysis that goes beyond the scope of this paper.

\begin{tabular}{lccc}
\hline & \multicolumn{3}{c}{ Area flooded $\left(\mathrm{km}^{2}\right)$} \\
\cline { 2 - 4 } & $(0,2.5]$ & $(2.5,10]$ & $(10,50]$ \\
\hline Flood category & Small & Medium & Large \\
No. observed events & 13 & 2 & 1 \\
Return period (yrs) & 5 & 35 & 70 \\
\hline
\end{tabular}

Table 8: Return period estimation. 


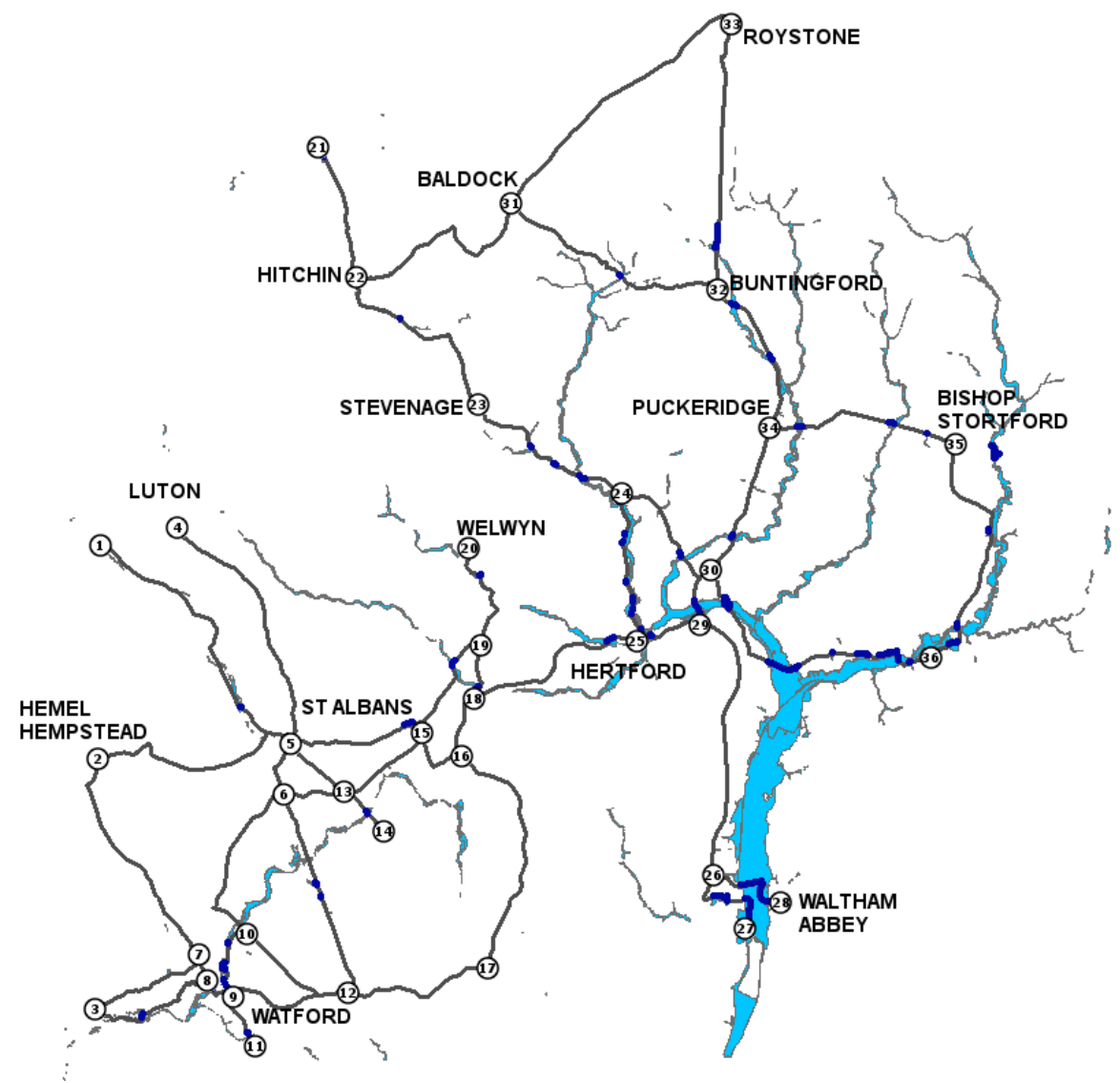

Figure 2: Hertfordshire A-road network.

For simplicity, we use the number of events in each category that occurred over the past 70 years to estimate return periods (see Table 8).

We consider all single arc protection plans. To this, we add some multiple arc plans based on a proximity criteria. The costs of implementing protection plans are assumed to be dependent on arc lengths in the same way as was done in Section 5 for the test instances. The protection budget is computed as a percentage of the resources needed to implement the highest level of protection for the entire network (equivalent to 9,825 protection units).

Table 9 reports how the shortest path cost decreases when the protection budget is increased. The first two increments (from $0 \%$ to $2 \%$ and from $2 \%$ to $4 \%$ ) are the most significant. Investing a budget of $10 \%$ results in a $26 \%$ reduction in the expected shortest path cost over the planning horizon.

\begin{tabular}{ccccccc}
\hline & \multicolumn{6}{c}{ Budget } \\
\cline { 2 - 7 } & $0 \%$ & $2 \%$ & $4 \%$ & $6 \%$ & $8 \%$ & $10 \%$ \\
\hline$\Delta$ Obj & $0 \%$ & $-8 \%$ & $-16 \%$ & $-21 \%$ & $-23 \%$ & $-26 \%$ \\
\hline
\end{tabular}

Table 9: Change in expected all-pairs shortest path cost $(\Delta \mathrm{Obj})$ versus budget. 


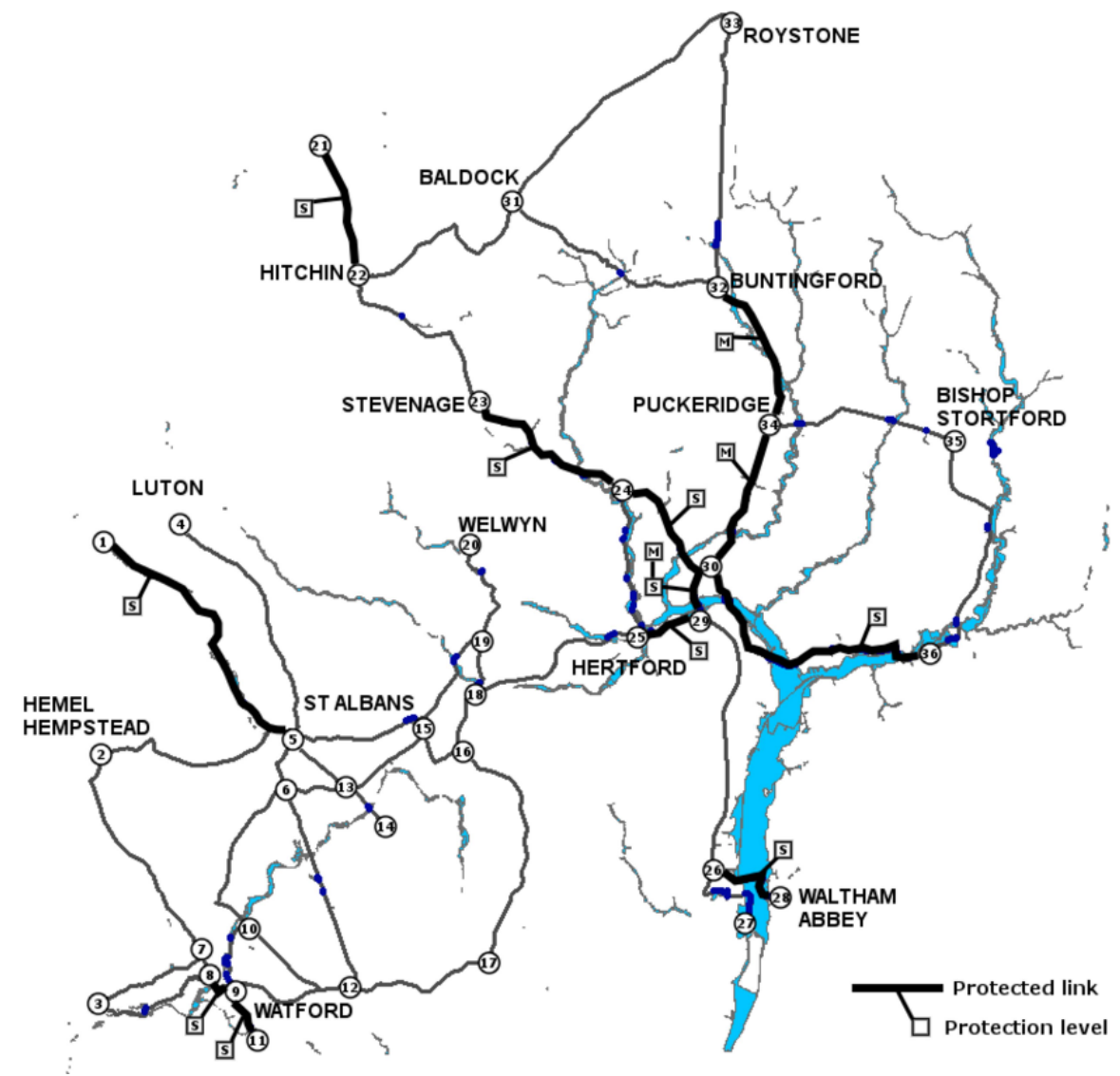

Figure 3: Selected protections given a protection budget of $10 \%$.

In Figure 3, we show the set of selected protection plans when the protection budget is equal to $10 \%$. The map shows, along with the protected links, the protection level of each arc. The majority of protections are designed to thwart small floods. No link is fortified at the highest possible level of protection. The results indicate that the central area of the network (close to the town of Hertford) is the most critical. Interestingly, arc 29-30 is initially protected at a small level in the first period and subsequently upgraded to a medium level in the last period.

Next, we compare the results of our base model with those obtained by adopting the probustness criteria introduced by Snyder and Daskin (2006). The p-robustness measure is combined with our model by adding the following set of constraints:

$$
\sum_{t=1}^{T} \sum_{k \in A} \sum_{o \in N}\left(d_{k} y_{k s t}^{o}+\Delta d_{k} \gamma_{k s t}^{o}\right) \leq(1+\mathrm{p}) D_{s}^{*} \quad \forall s \in S
$$

where $D_{s}^{*}$ is the objective value obtained by solving our base model with only scenario $s$ as input. 


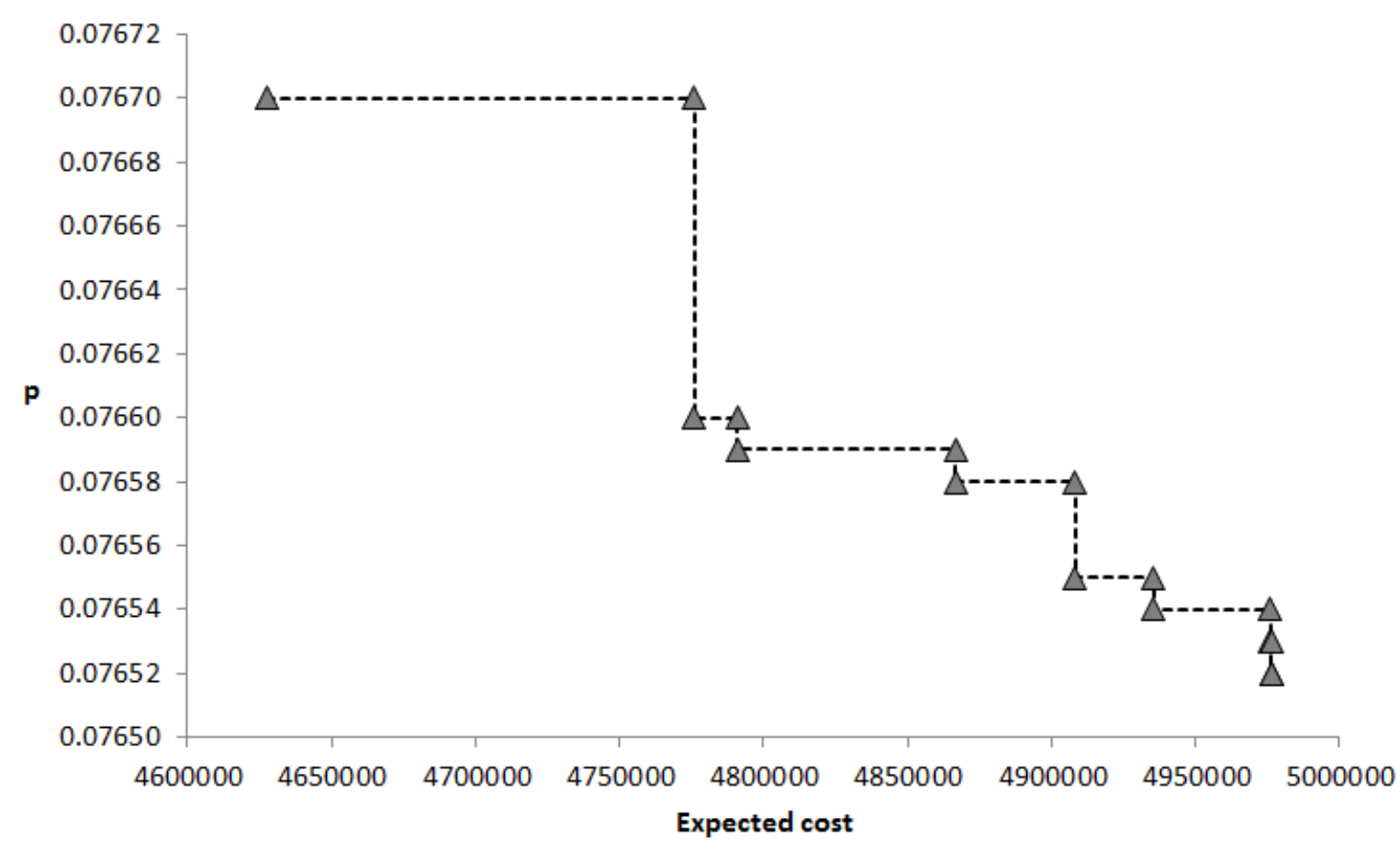

Figure 4: Maximum regret versus expected travel cost when the protection budget is equal to $5 \%$.

The results of this analysis are summarised in Figure 4 . The values of $\mathrm{p}$ are obtained following the same approach used by Snyder and Daskin (2006). We solve the problem with $\mathrm{p}=\infty$ and then set $\mathrm{p}$ to the maximum regret minus 0.00001 . Subsequently, $\mathrm{p}$ is decremented by 0.00001 until the problem becomes infeasible. Figure 4 shows that improvements in robustness are not justified by the costs. At best, a 0.00018 (0.23\%) decrease in relative regret (p) can be achieved for a $7.5 \%$ increase in expected travel cost when the protection budget is $5 \%$. Using higher budgets and or different values of $\mathrm{p}$ for different scenarios did not have any appreciable impact on robustness (results not reported).

We also carried out a sentivity analysis to understand how uncertainty in the estimation of return periods might impact optimal protection strategies. Specifically, we allowed the return period to vary by $\pm 20 \%$ from the base case for small, medium, and large floods, resulting in a total of 27 cases as shown in Table 10. The budget used in this analysis was set to $10 \%$.

Figure 5 shows the net percentage change in expected travel cost relative to a $0 \%$ protection budget. The figure clearly highlights that it is the return period of small floods that has the strongest impact on the objective value. In particular, the different flood scenarios clearly separate into three sub-categories (cases 1-9, cases 10-18, and cases 19-27) based entirely on the return period for small floods. Within these three sub-categories, only relatively small 


\begin{tabular}{|c|c|c|c|c|c|c|c|c|c|c|c|}
\hline \multirow[b]{2}{*}{ Case } & \multicolumn{3}{|c|}{ Return period (yrs) } & \multirow[b]{2}{*}{ Case } & \multicolumn{3}{|c|}{ Return period (yrs) } & \multirow[b]{2}{*}{ Case } & \multicolumn{3}{|c|}{ Return period (yrs) } \\
\hline & Small & Medium & Large & & Small & Medium & Large & & Small & Medium & Large \\
\hline 1 & 4 & 28 & 56 & 10 & 5 & 28 & 56 & 19 & 6 & 28 & 56 \\
\hline 2 & 4 & 28 & 70 & 11 & 5 & 28 & 70 & 20 & 6 & 28 & 70 \\
\hline 3 & 4 & 28 & 84 & 12 & 5 & 28 & 84 & 21 & 6 & 28 & 84 \\
\hline 4 & 4 & 35 & 56 & 13 & 5 & 35 & 56 & 22 & 6 & 35 & 56 \\
\hline 5 & 4 & 35 & 70 & 14 & 5 & 35 & 70 & 23 & 6 & 35 & 70 \\
\hline 6 & 4 & 35 & 84 & 15 & 5 & 35 & 84 & 24 & 6 & 35 & 84 \\
\hline 7 & 4 & 42 & 56 & 16 & 5 & 42 & 56 & 25 & 6 & 42 & 56 \\
\hline 8 & 4 & 42 & 70 & 17 & 5 & 42 & 70 & 26 & 6 & 42 & 70 \\
\hline 9 & 4 & 42 & 84 & 18 & 5 & 42 & 84 & 27 & 6 & 42 & 84 \\
\hline
\end{tabular}

Table 10: List of different return period value permutations for small, medium, large floods.

changes in the objective value are observed depending on the return periods for medium and large floods. For example, increasing the return period of small floods from just 4 to 5 years (the equivalent of going from a 0.25 to a 0.2 chance of occuring in any given year), causes net expected travel cost to drop by more than 2 -fold, with values in the range $[-9 \%,-12 \%]$ versus $[-25 \%,-28 \%]$, respectively. What this suggests is that errors in return periods estimates for medium and large floods are of less concern compared to small floods.

Finally, we evaluate the robustness of optimal protection strategies to uncertainty in flood return periods. Table 11 reports the percentage increase in the objective function for the base case solution (i.e., case 14) across all 27 permutations for small, medium, and large

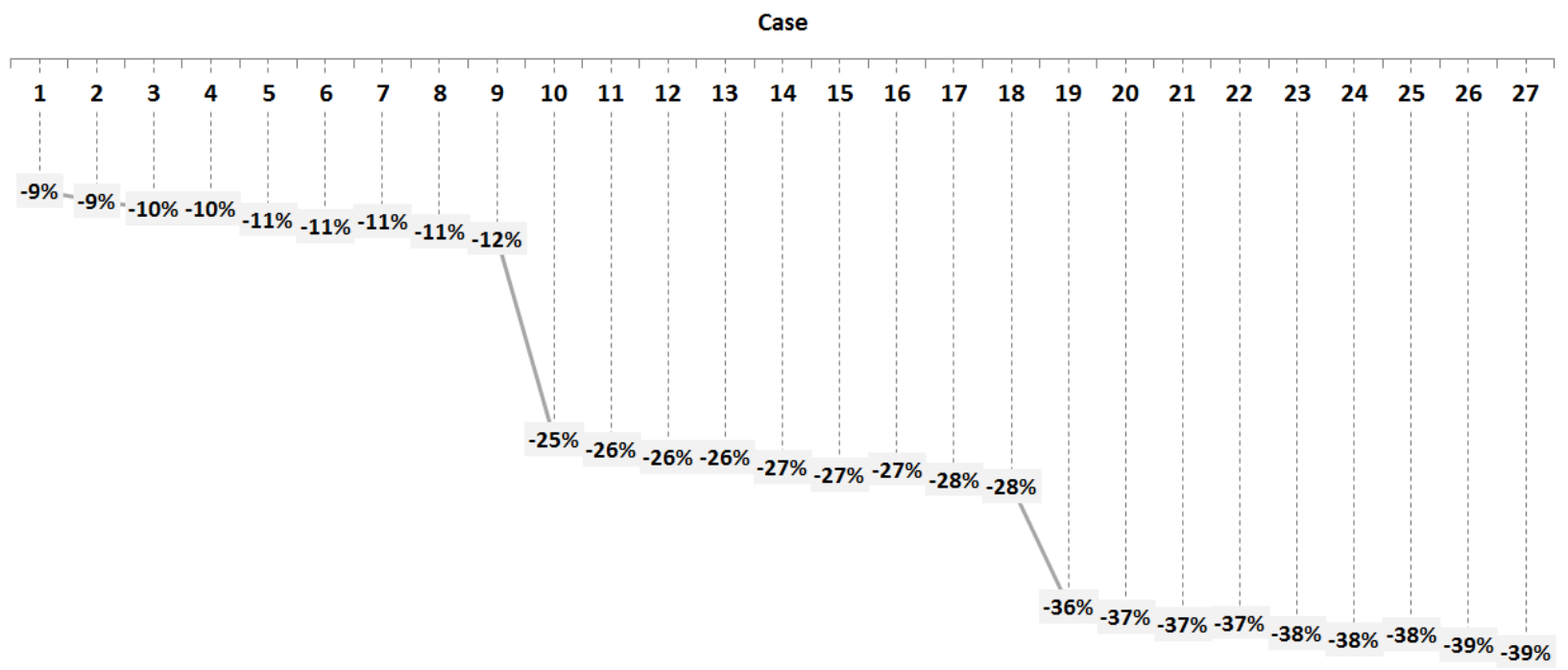

Figure 5: Net percentage change in expected travel cost give a protecton budget of $10 \%$. 
flood return period values. The table indicates that base case optimal solution is highly robust even when the estimation error for return periods is as high as $20 \%$. Indeed, the base case solution produced no increase in expected travel cost for 21 out the 27 cases. In the remaining 6 cases, the increase in cost was marginal (i.e., under $0.3 \%$ ).

\begin{tabular}{cc|cc|cc}
\hline Case & Inc $(\%)$ & Case & Inc $(\%)$ & Case & Inc $(\%)$ \\
\hline $\mathbf{1}$ & $0.00 \%$ & $\mathbf{1 0}$ & $0.00 \%$ & $\mathbf{1 9}$ & $0.17 \%$ \\
$\mathbf{2}$ & $0.00 \%$ & $\mathbf{1 1}$ & $0.00 \%$ & $\mathbf{2 0}$ & $0.24 \%$ \\
$\mathbf{3}$ & $0.00 \%$ & $\mathbf{1 2}$ & $0.04 \%$ & $\mathbf{2 1}$ & $0.29 \%$ \\
$\mathbf{4}$ & $0.00 \%$ & $\mathbf{1 3}$ & $0.00 \%$ & $\mathbf{2 2}$ & $0.00 \%$ \\
$\mathbf{5}$ & $0.00 \%$ & $\mathbf{1 4}$ & $0.00 \%$ & $\mathbf{2 3}$ & $0.00 \%$ \\
$\mathbf{6}$ & $0.00 \%$ & $\mathbf{1 5}$ & $0.00 \%$ & $\mathbf{2 4}$ & $0.00 \%$ \\
$\mathbf{7}$ & $0.00 \%$ & $\mathbf{1 6}$ & $0.00 \%$ & $\mathbf{2 5}$ & $0.00 \%$ \\
$\mathbf{8}$ & $0.01 \%$ & $\mathbf{1 7}$ & $0.00 \%$ & $\mathbf{2 6}$ & $0.00 \%$ \\
$\mathbf{9}$ & $0.03 \%$ & $\mathbf{1 8}$ & $0.00 \%$ & $\mathbf{2 7}$ & $0.00 \%$ \\
\hline Avg & $0.01 \%$ & & $0.00 \%$ & & $0.08 \%$ \\
\hline
\end{tabular}

Table 11: Robustness of the base case optimal solution to flood return period uncertainty.

\section{Conclusions}

In this paper, we introduce a scenario-based model to identify the optimal set of plans to adopt for protecting a road network against flooding. When dealing with flood protection, a large number of measures may be under consideration. These measures can differ significantly in terms of cost and the level of protection provided. We incorporate these aspects by considering different design safety standards and using protection plans involving multiple road links as opposed to single road links. A multi-period planning horizon is adopted to take into account the possibility that protection resources are distributed over time. The consequences of disruptions are estimated in terms of the expected all-pairs shortest path travel cost.

We find that solving the problem with a general purpose solver is challenging. A GRASP heuristic is introduced to solve realistic sized problem instances. Tests on randomly generated networks of different size demonstrate the efficiency of the heuristic. Finally, a case study of the Hertfordshire A-road network provides some useful insights regarding road infrastructure protection planning in the face of flood risk.

Future work might focus on the incorporation of user-equilibrium constraints so that the relationship between travel time and traffic flow is more accurately captured. Such a model would invariably be considerably harder to solve, thus necessitating the need to devise efficient 
and scalable solution approaches. A further extension of our model might be to consider using fragility curves to characterise the probability that a protection measure fails (e.g., due to overtopping). It would also be interesting to consider partial protection of road links. Finally, future research might focus on the development of integrated models that combine protection of urban areas, rural areas, and roads or other infrastructure.

\section{Acknowledgements}

We thank the associate editor and an annoymous referee for helpful comments provided on an earlier draft of the paper.

\section{References}

Alderson D L, Brown G G, Carlyle W M, and Wood R K (2011). Solving defender-attackerdefender models for infrastructure defense. Pp. 28-49 in: Wood, R K and Dell R F (Eds.) Operations research, computing, and homeland defense. INFORMS, Hanover (MD).

Alguacil N, Delgadillo A, and Arroyo J M (2014). A trilevel programming approach for electric grid defense planning. Computers \& Operations Research 41: 282-290.

Aliakbarian N, Dehghanian F, and Salari M (2015). A bi-level programming model for protection of hierarchical facilities under imminent attacks. Computers $\& 3$ Operations Research 64: $210-224$.

Baghalian A, Rezapour S, and Farahani R Z (2013). Robust supply chain network design with service level against disruptions and demand uncertainties: A real-life case. European Journal of Operational Research 227(1): 199-215.

Ben-Tal A, Do Chung B, Mandala S R, and Yao T (2011). Robust optimization for emergency logistics planning: Risk mitigation in humanitarian relief supply chains. Transportation Research Part B: Methodological (8): 1177-1189.

Berman O, Krass D, and Menezes M B (2007). Facility reliability issues in network p-median problems: strategic centralization and co-location effects. Operations Research 55(2): 332350.

Cappanera P, and Scaparra M P (2011). Optimal allocation of protective resources in shortestpath networks. Transportation Science 45(1): 64-80. 
Carrington D and Weaver M (2014). Emergency funding to repair damaged UK flood defences raised to $£ 130 \mathrm{~m}$. The Guardian 6 Feb 2014. Available at: http://www . theguardian . com/ environment/2014/feb/06/emergency-funding-uk-flood-defences-storms-weather [Accessed 10 Nov 2015]

Chang S E (2003). Transportation planning for disasters: an accessibility approach. Environment and Planning A 35(6): 1051-1072.

Chen G, Daskin M S, Shen Z J M, and Uryasev S (2006). The $\alpha$-reliable mean-excess regret model for stochastic facility location modeling. Naval Research Logistics 53(7): 617-626.

Church R L and Scaparra M P(2007). Protecting critical assets: The r-interdiction median problem with fortification. Geographycal Analysis 39(2): 129-146.

Current J, Ratick S, and ReVelle C (1998). Dynamic facility location when the total number of facilities is uncertain: A decision analysis approach. European Journal of Operational Research 110(3): 597-609.

Du L, and Peeta S (2014). A stochastic optimization model to reduce expected post-disaster response time through pre-disaster investment decisions. Networks and Spatial Economics 14(2): 271-295.

Dutta D, Herath S, and Musiake K (2003). A mathematical model for flood loss estimation. Journal of Hydrology 277(1): 24-49.

Environmental Agency (2009) Flooding in England: A National Assessment of Flood Risk. Available at: https://www.gov.uk/government/uploads/system/uploads/ attachment_data/file/292928/geho0609bqds-e-e.pdf [Accessed 10 Nov 2015]

Fan Y, and Liu C (2010). Solving stochastic transportation network protection problems using the progressive hedging-based method. Networks and Spatial Economics 10(2): 193-208.

Faturechi R, and Miller-Hooks E. (2014). Travel time resilience of roadway networks under disaster. Transportation Research Part B: Methodological 70: 47-64.

Feo T A, and Resende M G (1995). Greedy randomized adaptive search procedures. Journal of Global Optimization 6(2): 109-133.

Floyd R W (1962). Algorithm 97: shortest path. Communications of the ACM 5(6): 345.

Görmez N, Köksalan M, and Salman F S (2011). Locating disaster response facilities in Istanbul. Journal of the Operational Research Society 62(7): 1239-1252. 
Gumbel E J (1941). The return period of flood flows. The Annals of Mathematical Statistics 12(2): 163-190.

He X, and Liu H X (2012). Modeling the day-to-day traffic evolution process after an unexpected network disruption. Transportation Research Part B: Methodological 46(1): 50-71.

Huang Y, Fan Y, and Cheu R (2007). Optimal allocation of multiple emergency service resources for protection of critical transportation infrastructure. Transportation Research Record: Journal of the Transportation Research Board (2022) 1-8.

Ishfaq R (2013). Intermodal shipments as recourse in logistics disruptions. Journal of the Operational Research Society 64(2): 229-240.

Jin J G, Lu L, Sun L, and Yin J (2015). Optimal allocation of protective resources in urban rail transit networks against intentional attacks. Transportation Research Part E: Logistics and Transportation Review 84: 73-87.

Jozefowiez N, Mancel C, and Mora-Camino F (2012). A heuristic approach based on shortest path problems for integrated flight, aircraft, and passenger rescheduling under disruptions. Journal of the Operational Research Society 64(3): 384-395.

Liberatore F, Scaparra M P, and Daskin M S (2011). Analysis of facility protection strategies against an uncertain number of attacks: the stochastic R-interdiction median problem with fortification. Computers \& Operations Research 38(1): 357-366.

Liu C, Fan Y, and Ordonez F (2009). A two-stage stochastic programming model for transportation network protection. Computers $\&$ Operations Research 36(5): 1582-1590.

Losada C, Scaparra M P, and O'Hanley J R (2012). Optimizing system resilience: a facility protection model with recovery time. European Journal of Operational Research 217(3): 519-530.

Luo T, Maddocks A, Iceland C, Ward P, and Winsemius H (2015). World's 15 Countries with the Most People Exposed to River Floods. World Resources Institute. Available at: http://www.wri.org/blog/2015/03/world \\%E2\\%80\ \%99s-15-countries-most-people-exposed-river-floods [Accessed 10 Nov 2015]

Madadi A, Kurz M E, Taaffe K M, Sharp J L, and Mason S J (2014). Supply network design: Risk-averse or risk-neutral? Computers 6 Industrial Engineering 78: 55-65. 
Najafi M, Eshghi K, and Dullaert W (2013). A multi-objective robust optimization model for logistics planning in the earthquake response phase. Transportation Research Part E: Logistics and Transportation Review 49(1): 217-249.

Peeta S, Salman F S, Gunnec D, and Viswanath K. (2010). Pre-disaster investment decisions for strengthening a highway network. Computers $\& 3$ Operations Research 37(10): 17081719 .

Peng P, Snyder L V, Lim A, and Liu Z (2011). Reliable logistics networks design with facility disruptions. Transportation Research Part B: Methodological 45(8): 1190-1211.

Rawls C G, and Turnquist M A (2010). Pre-positioning of emergency supplies for disaster response. Transportation Research Part B: Methodological 44(4): 521-534.

Rawls C G, and Turnquist M A (2012). Pre-positioning and dynamic delivery planning for short-term response following a natural disaster. Socio-Economic Planning Sciences 46(1) $46-54$.

Sarhadi H, Tulett D M, and Verma M (2015). A defender-attacker-defender approach to the optimal fortification of a rail intermodal terminal network. Journal of Transportation Security 8(1-2) 17-32.

Snyder L V., and Daskin M S (2006). Stochastic p-robust location problems. IIE Transactions 38(11): 971-985.

Sohn J (2006). Evaluating the significance of highway network links under the flood damage: An accessibility approach. Transportation Research Part A: Policy and Practice 40(6): 491-506.

Soyster A L (1973). Technical note?convex programming with set-inclusive constraints and applications to inexact linear programming. Operations Research 21(5): 1154-1157.

Starita S, and Scaparra M P (2016). Optimizing dynamic investment decisions for railway systems protection. European Journal of Operational Research 248(2): 543-557.

Suarez P, Anderson W, Mahal V, and Lakshmanan T R (2005). Impacts of flooding and climate change on urban transportation: A systemwide performance assessment of the Boston Metro Area. Transportation Research Part D: Transport and Environment 10(3): 231-244.

Ukkusuri S V, and Yushimito W F (2009). A methodology to assess the criticality of highway transportation networks. Journal of Transportation Security 2(1-2): 29-46. 
Viswanath K, Peeta S, and Salman S F (2004). Investing in the links of a stochastic network to minimize expected shortest path. Purdue University Economics Working Papers, Purdue University, Department of Economics.

Yin Y (2008). A scenario-based model for fleet allocation of freeway service patrols. Networks and Spatial Economics 8(4) 407-417.

Zhang X, Zheng Z, Zhu Y, and Cai, K. Y. (2014). Protection issues for supply systems involving random attacks. Computers \& Operations Research 43: 137-156 